\title{
An unusual localization of genital ulcer in Behçet's disease: external urethral meatus
}

\author{
Cagri Turan ${ }^{1}$ and İbrahim Karabulut ${ }^{1}$ \\ ${ }^{1}$ Erzurum Training and Research Hospital
}

March 10, 2021

\section{An unusual localization of genital ulcer in Behçet's disease: external urethral meatus}

Running head: Unusual genital ulcer in Behçet's disease

Çağrı Turan ${ }^{1}$, İbrahim Karabulut ${ }^{2}$

${ }^{1}$ Department of Dermatology and Venereology, the Republic of Turkey, Health Sciences University Erzurum Regional Training and Research Hospital, Turkey

${ }^{2}$ Department of Urology, the Republic of Turkey, Health Sciences University Erzurum Regional Training and Research Hospital, Turkey

Corresponding Author : Çağrı TURAN; Department of Dermatology and Venereology, the Republic of Turkey, Health Sciences University Erzurum Regional Training and Research Hospital, Turkey; Üniversite Mahallesi, Çat Yolu Cd., Yakutiye/Erzurum, Post Code: 25070

e-mail: cagrituranmd@gmail.com, telephone number: +905445252504

Word count: 471

Table count: 0

Supplementary table: 0

Figure count: 1

Funding sources : We declare no financial support or relationships that may pose a conflict of interest.

Conflict of interest: There is no conflict of interest.

The paper has not been published or submitted for publication elsewhere.

All authors have contributed significantly, and all authors agree with the content of the manuscript.

Informed consent form was obtained from the patient.

Key words: Behçet's disease, genital ulcer, Urethra, Vasculitis

Dear editor,

Behçet's disease (BD) is a chronic, recurrent, multisystemic vasculitis which can affect all vascular system. The most common symptom is genital ulcer accompanying oral aphthae, and its diagnosis is currently made according to the International Criteria for Behçet's Disease (ICBD). Providing two points for oral aphthae, genital ulcers, and ocular involvement and one point for the other skin lesions (erythema nodosum, papulopustular/acneiform lesions in post-adolescent), vascular involvement, and neurological findings are evaluated if the patient scores reach four or more; the patient is considered to be $\mathrm{BD}^{1,2}$. In men, genital 
aphthous ulcers occur in 60 to $65 \%$ of cases and are most common in the scrotum, shaft and glans penis, and rarely in the groin and perineum, extremely rare in the urethral orifice ${ }^{2,3}$

We presented a patient with a complaint of painful micturition, ultimately diagnosed with BD. A 34-year-old male patient was consulted from the urology following the evaluation of his painful voiding complaint 10 days accompanying wound in the periurethral orifice. On genital examination, an oval, and sharp circumscribed aphthous ulcer with a serous floor, approximately $4 \mathrm{~mm}$ in diameter, was seen on the external urethral orifice (Figure 1a). The patient who had no known disease has refused to use any medication, similar complaints, suspicious sexual contact, except for oral aphthae recurring 8-10 times a year (Figure 1b). After noticing a few pustular lesions with peripheral partial erythema on his back, we focused on BD and inflammatory bowel diseases in the examination (Figure 1c). We learned that the patient had no family history and other related symptoms. Bowel habits were normal. Hepatitis, HIV, syphilis serologies were negative. CRP and sedimentation were $3.2 \mathrm{mg} / \mathrm{dl}(0-5 \mathrm{mg} / \mathrm{dl})$ and $27 \mathrm{~mm} /$ hour $(0-20 \mathrm{~mm} /$ hour $)$; respectively. Other hematological and biochemical parameters were within normal limits. Complement levels, antinuclear antibody, anti-dsDNA, p-ANCA, c-ANCA and rheumatoid factor were normal. Pathergy test was positive. The patient was diagnosed with BD with a score of 6 according to the current ICBD, and no pathology was found in the eye and cardiology consultations. His complaints improved within 1 week without leaving any scar, after treatment with topical betamethasone valerate applied to the urethral orifice and oral colchicine $1.5 \mathrm{mg} /$ day. No clinical progression has been observed for 2 years in the follow-up.

A urethral ulcer is an unusual finding in BD. Aktaş recently reported a 27-year-old male patient with clinical features quite similar to our case, but with negative pathergy ${ }^{4}$. Interestingly, Cobilinschi et al. reported that a 34-year-old female patient with BD who was admitted with progressive dysuria, pain in the right lumbar region, and hydronephrosis was determined a necrotic ulcer in the ureter whose histopathology was compatible with vasculitis ${ }^{5}$.

We present this case to draw attention to the aphthous ulcer in the urethra as an unusual genital involvement for $\mathrm{BD}$, and the importance of physical examination.

\section{References:}

1. Davatchi F, Assaad-Khalil S, Calamia K, et al: The International Criteria for Behçet's Disease (ICBD): A collaborative study of 27 countries on the sensitivity and specificity of the new criteria.J Eur Acad Dermatol Venereol 2014;28(3):338-47.

2. Alpsoy E, Zouboulis CC and Ehrlich GE: Mucocutaneous lesions of Behcet's disease. Yonsei Med $J$ 2007;48(4):573-85.

3. Altenburg A, Papoutsis N, Orawa H, Martus P, Krause L and Zouboulis CC: [Epidemiology and clinical manifestations of Adamantiades-Behçet disease in Germany - current pathogenetic concepts and therapeutic possibilities]. J Dtsch Dermatol Ges 2006;4(1):49-64.

4. Aktaş H: An extraordinary manifestation of Behçet's disease in a young male: Urinary meatus ulcer. Int J Rheum Dis2020;23(2):271-72.

5. Cobilinschi C, Belinski C and Opris-Belinski D: Rare case of female Behçet's disease with urological involvement. Rom J Rheumatol2019;28(2):80-83.

\section{Figure Legend:}

Figure 1. a: an aphthous ulcer with a serous base, oval and sharply circumscribed, about $4 \mathrm{~mm}$ in diameter, in the left lateral aspect of the external urethral orifice; b: oral aphthae; c: a pustule with peripheral, partial erythema on the back 


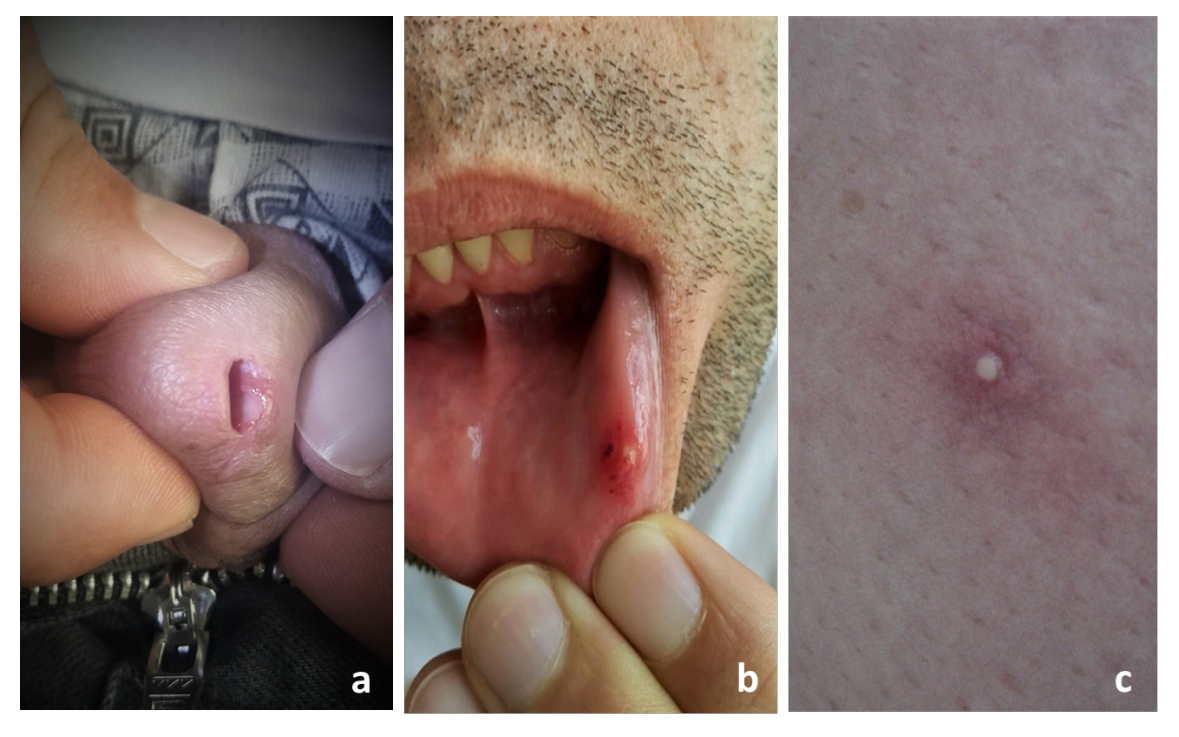

